\title{
Primi sondaggi sul testimone J del volgarizzamento catalano della Consolatio Philosophiae*
}

\author{
A preliminary survey of $\mathrm{ms}$. J \\ of the Catalan translation of the Consolatio Philosophiae
}

\author{
Chiara Concina \\ chiara.concina@univr.it \\ Università degli Studi di Verona
}

Riassunto: La vicenda testuale del volgarizzamento catalano del De consolatione philosophiae di Boezio si caratterizza per la problematicità dei suoi aspetti redazionali e per la complessità della sua tradizione manoscritta. Il perduto testo originale di questa traduzione, realizzata dal frate domenicano Pere Saplana in un periodo compreso tra il 1358 e il 1362, si è infatti conservato in due redazioni differenti. La prima $(\alpha)$, anonima, è tramandata da un testimone completo in castigliano e da un frammento catalano. La seconda $(\beta)$, tràdita da un numero elevato di testimoni, è invece il risultato di un lavoro di revisione operato sul testo di Saplana dal domenicano Antoni Ginebreda (1390 c.). In tempi recenti uno dei due codici del Boeci conservati presso l'Arxiu Comarcal de la Segarra di Cervera (sigla J) è stato indicato come possibile latore di una redazione prossima ad $\alpha$, considerata in molti punti quella più conservativa rispetto all'originale di Saplana. Il contributo offre un'indagine preliminare riguardante la struttura e i contenuti del testo tràdito dal codice $\mathrm{J}$ ponendolo in relazione con quanto tramandato da $\alpha$ e $\beta$.

Parole-chiave: Boezio; volgarizzamenti medievali; volgarizzamenti catalani; Pere Saplana; Antoni Ginebreda

\begin{abstract}
The history of Boethius's De consolatione philosophiae Catalan translation is particularly complex for what concerns its manuscript tradition as well as for the textual differences that can be found in the exstant versions of it. The lost original version of this vernacular translation, written around the years 1358-1362 by the Dominican friar Pere Saplana, is preserved in two different versions. The first one $(\alpha)$ is anonymous, and has survived in its complete form in a Castilian translation and in a Catalan fragment. The second $(\beta)$ is transmitted by a large number of witnesses and is the result of a revision of Saplana's text made around 1390 by the Dominican Antoni Ginebreda. One of the two manuscripts containing this translation preserved in the Arxiu Comarcal de la Segarra of Cervera (designed as J) was recently mentioned as the possible bearer of a version very similar to $\alpha$, considered the closest to Saplana's original text. The paper offers a first analysis of the structure and the readings of the text of $\mathrm{J}$, comparing them to the versions transmitted by $\alpha$ and $\beta$.
\end{abstract}

Keywords: Boethius; Medieval Translations; Catalan Translations; Pere Saplana; Antoni Ginebreda

DATA PRESENTACIÓ: 23/04/2015 ACCEPTACIÓ: 12/05/2015 · PUBLICACIÓ: 15/06/2015

SCRIPTA, Revista internacional de literatura i cultura medieval i moderna, núm. 5 / juny 2015 / pp. 182 - 206 ISSN: 2340-4841 · doi:10.7203/SCRIPTA.5.6390 


\section{Premessa}

La tradizione manoscritta del più antico volgarizzamento catalano della Consolatio Philosophiae (che è forse anche il primo del dominio iberico) presenta un problema di fondo che riassumo qui brevemente. Di questo testo in prosa, che è in molti punti una traduzione del commento latino alla Consolatio Philosophiae di Guglielmo d'Aragona (seconda metà del XIII secolo), ${ }^{1}$ sono note due differenti redazioni che risalirebbero entrambe a un unico testo-modello, databile al 1358-1362 c. e ascritto al frate domenicano Pere Saplana che lo dedicò all'infante Giacomo IV di Maiorca. ${ }^{2}$ L'attribuzione a Saplana si evince dal prologo di un perduto codice proveniente dall'abbazia di Montserrat, di cui si conserva copia unicamente in una trascrizione eseguita nel 1919 da padre Jaume Pasqual (Villanueva 1851: 205-206; Albareda 1919 [1920]: 198-200; Riera i Sans 1984: 298299).

La prima delle due redazioni $(\alpha)$ che sarebbero derivate dal modello costituito dal volgarizzamento di Saplana, è anonima ed è conservata dal frammento catalano di cinque carte del ms. Ripoll 113 (Barcelona, Archivo de la Corona de Aragón $=\mathrm{R})^{3}$ e dalla traduzione castigliana completa contenuta nel ms. 10193 della Biblioteca Nacional de Madrid $(=\mathrm{N}) \cdot{ }^{4}$ In tempi recenti il codice senza segnatura dell'Arxiu Comarcal de la Segarra di Cervera $(=\mathrm{J})$, sul quale si ritornerà a breve, è stato segnalato come latore di un testo accostabile a quello tràdito da $\alpha$.

La seconda redazione $(\beta)$ sarebbe invece opera di un altro domenicano, Antoni Ginebreda, ${ }^{5}$ che

\footnotetext{
* Sono profondamente grata a Francesca Ziino che con grande generosità mi ha inviato una copia digitale della sua tesi di dottorato, che si è rivelata indispensabile nell'affrontare il testo di J. Ringrazio Carmen Olmedilla Herrero, che mi ha permesso di consultare la sua edizione del commento latino di Guglielmo d'Aragona. La mia gratitudine va anche a Klaus Kempf, per il suo impagabile aiuto con il Cod. Hisp. 145, e al personale dell'Arxiu Comarcal de la Segarra, per la professionalità e la disponibilità con le quali mi hanno inviato le riproduzioni dei due codici conservati a Cervera.
}

1 Edito in Olmedilla Herrero (ed.) 1997, che sostituisce il precedente lavoro di Terbille (ed.) 1972. La dimostrazione che il volgarizzamento catalano dipende dal commento la

tino di Guglielmo è data in González Rolan / Saquero Suárez-Somonte 1992. Ziino 2007 ha poi notato che, oltre al commento di Guglielmo, nella traduzione catalana si innervano anche materiali esegetici di provenienza differente.

2 Per Saplana cfr. Coll 1935.

3 Il frammento occupa le cc. 65a-69d del codice (il frammento è disponibile online su PARES - Portal de Archivos Españoles: http://www.mcu.es/archivos/MC/ACA/BasesDatos.html) ed è stato trascritto in Bofarull y Mascaró ed. 1857: 395-413, poi edito in Ziino ed. 2007, 2: 176-197. Le sigle utilizzate per designare i manoscritti sono quelle proposte in Doñas 2007.

4 Edito in Ziino ed. 1997, 2: 1-175, ma per cui cfr. anche Ziino 1998 e 2003. Il colophon di questo codice reca il nome del copista (Pedro de Valladolid), il luogo (Alcañiz) e la data (21 settembre 1436). Per una descrizione di R e di N si consultino Ziino 1997, 1: 53-58 e Doñas 2007: 299 e 301.

5 Per i dati documentari relativi a Ginebreda si vedano Coll 1949: 16-31 e De Puig i Oliver 2000.

SCRIPTA, Revista internacional de literatura i cultura medieval i moderna, núm. 5 / juny 2015 / pp. 182 - 206 
verso la fine del XIV secolo (1390 c.) rimaneggiò, su richiesta del cavaliere valenciano Bernat Joan, il testo di Saplana ${ }^{6}$, rivedendolo e integrandolo in alcuni punti, mediante aggiunte la cui fonte principale è da individuarsi nel commento di Nicholas Trevet $(1300$ c. $){ }^{7}$ I testimoni latori di questa versione contengono in alcuni casi, ma non sempre, una rubrica iniziale che attribuisce il lavoro di traduzione a Ginebreda e un prologo in cui il traduttore/revisore afferma di aver lavorato su un testo già esistente dedicato all'infante Giacomo di Maiorca, apportandovi alcuni miglioramenti e aggiungendo la traduzione di passi della Consolatio che erano stati omessi o che risultavano insoddisfacenti nel suo modello (per cui cfr. infra $\$ 5) .{ }^{8}$ Il volgarizzamento di Ginebreda è tràdito da 9 testimoni manoscritti catalani, ${ }^{9}$ un incunabolo catalano, ${ }^{10}$ e infine da due manoscritti ${ }^{11}$ e quattro stampe castigliane. ${ }^{12}$ A completamento del quadro si aggiungano anche una ritraduzione latina del

6 Non così Keightley 1987: 182-184, per cui la redazione $\alpha$ sarebbe deriverebbe da un rimaneggiamento di $\beta$ e sarebbe dunque da considerare ad esso posteriore.

7 Il commento del Trevet è disponibile online nell'edizione (rimasta dattiloscritta) curata da Edmund Silk: Expositio Fratris Nicolai Trevethi Anglici Ordinis praedicatorum super Boetio De Consolatione: $<$ http://minnis.commons.yale.edu/>.

8 Per le ipotesi riguardanti la quantità e la natura degli interventi effettuati da Ginebreda sulla prima redazione cfr. Riera i Sans 1984: 315-316; González Rolan / Saquero Suárez-Somonte 1992: 333; Ziino ed. 1997, 1: 142-155.

9 B = Barcelona, Biblioteca Universitària, ms. 77, sec. XV (Muntaner ed. 1873; il ms. è consultabile online sul sito della Biblioteca virtual Miguel de Cervantes: http://www.cervantesvirtual.com/obra-visor/de-consolacio/visor/); C = Barcelona, Biblioteca de Catalunya, ms. 68, sec. XV; H = Munich, Bayerische StaatsBibliothek, Cod. Hisp. 145, sec. XV; K = Berkeley, Bancroft Library, ms. UCB 160, 1470-1480 c. (consultabile online: http://sunsite.berkeley.edu/cgibin/ebind2html/catalan/boethius?cap); $\mathrm{M}=$ Madrid, Biblioteca Nacional, ms. 18396, sec. XV; P = Paris, Bibliothèque nationale de France, ms. Esp. 474, sec. XV; V = Avignon, Archive du Département de Vaucluse, fond Requin, ms. 177, sec. XV; Y = Cervera, Arxiu Comarcal de la Segarra, senza segnatura, 1426-1450 c.; Z = Sevilla, Biblioteca Capitular (Colombina), ms. 5-5-26, sec. XIV. Per la descrizione di questi codici si rinvia a Ziino 1997, 1: 38-52, da integrare con Doñas 2007 e Perarnau i Espelt 2006 (per il ms. H).

$10 \mathrm{~L}$ = Lérida, Heinric Botel, 2 giugno 1489 (editio princeps); di questa edizione si conosce un solo esemplare conservato a Madrid, Biblioteca Nacional, I-1280, cfr. Doñas 2007, 302 e l'Incunabula Short Tittle Catalogue = ISTC ib00811700 (online: http://www.bl.uk/catalogues/istc/).

11 Q = Madrid, Biblioteca Nacional, ms. II-589, sec. XV (disponibile online: http://fotos.patrimonionacional.es/ biblioteca/ibis/pmi/II_00589/index.html); D = Madrid, Real Biblioteca, ms. 8320, sec. XV, per cui cfr. ancora Doñas 2007: 301-302.

12 Le stampe castigliane si dividono in due gruppi, latori di due traduzioni che derivano da due diversi modelli catalani. Il primo gruppo è rappresentato unicamente dall'incunabolo $\mathrm{T}=$ Toulouse, Heinrich Mayer, 4 luglio 1488 (7 esemplari censiti in ISTC, ib00817000; l'ed. è consultabile online: http://bdh-rd.bne.es/viewer.vm?id=0000179078\&page=1); mentre il secondo raggruppamento comprende $\mathrm{Se}_{1}=$ Sevilla, Meinardo Ungut e Estanislao Polono, 18 febbraio 1497 (7 esemplari censiti in ISTC, ib00818000; consultabile in formato digitale sul sito della Biblioteca virtual Miguel de Cervantes: http://www.cervantesvirtual.com/obra/de-la-consolacion-de-la-filosofia/); $\mathrm{Se}_{2}=$ Sevilla, Meinardo Ungut e Estanislao Polono, 14 ottobre 1499; To = Toledo, Juan Varela de Salamanca, 15 ottobre 1511 (consultabile online sul sito http:// bvpb.mcu.es $/$ es $/$ consulta/busqueda referencia.cmd?posicion $=2 \&$ idValor $=53111 \&$ forma $=$ ficha\&id $=380) . \mathrm{Se}_{2}$ e To sono ristampe di $\mathrm{Se}_{1}$. 
testo di Ginebreda ${ }^{13}$ (Ziino 2001b) e un volgarizzamento ebraico realizzato intorno al 1412 (Zonta $2010)^{14}$, che presenta elementi riconducibili sia ad $\alpha$ che a $\beta$.

Tra i testimoni segnalati di recente (non inclusi dunque negli studi ad oggi disponibili sull'insieme della tradizione) e ancora poco sondati interesserà qui il manoscritto dell'Arxiu Comarcal de la Segarra (che si aggiunge a quello già noto $-\mathrm{Y}$ - conservato presso questa istituzione). A questo codice senza segnatura Antonio Doñas (2007: 298) ha attribuito la sigla J, includendolo, senza ulteriori precisazioni, tra i latori della redazione $\alpha$. Il manufatto è stato censito nel database online BITECA (Bibliografia de textos antics catalans, valencians $i$ balears) ${ }^{15}$ in cui viene fornita una scheda descrittiva curata da Gemma Avenoza (manid 2780), accompagnata dalla nota che segue: «podria tractar-se del mateix text que el que es conserva fragmentari a l'Arxiu de la Corona d'Aragó Ripoll, 113». Si tenterà dunque di fornire un'analisi preliminare relativa alla partitura interna, alla struttura e alle lezioni caratteristiche del testo tràdito da $\mathrm{J}$, mettendole in relazione sia con $\mathrm{N}$ (e laddove possibile con $\mathrm{R}$ ), che con le lezioni fissate nei codici della redazione $\beta .{ }^{16}$

\section{Il codice J (Cervera, Arxiu Comarcal de la Segarra, sin signatura, olim A)}

\subsection{Descrizione}

Riprendo, con qualche integrazione, la descrizione del manoscritto già data da Doñas e Avenoza:

Cervera, Arxin Comarcal de la Segarra, ms. senza segnatura, olim $A$

Codice cartaceo, acefalo e lacunoso, della fine del sec. XIV o dell'inizio del XV (1391?-1430?) di 290x190 mm. I richiami al centro del margine inferiore delle cc. XVv, XXXIV, LXIv, LXXVIIV, XCIIIV

13 London, British Library, mss. Harley 4335-4339, sec. XV; il codice contiene inoltre anche una versione con glosse di un volgarizzamento francese della Consolatio, per cui cfr. Cropp 1982-1983: 303-306 e Galderisi (dir.), 2/1: 384-385.

14 Il testo ebraico è tràdito da un testimone completo San Pietroburgo, Biblioteca dell'Istituto di Orientalistica dell'Accademia delle Scienze di Russia, MS B 18; e da un frammento: Città del Vaticano, Biblioteca Apostolica Vaticana, Neofiti 8, cc. 71v-82v.

15 Cfr.: http://bancroft.berkeley.edu/philobiblon/index.html. Il codice è segnalato anche nel database TRANSLAT, Traduccions al català medieval fins a 1500 (TCM): http://www.translatdb.narpan.net/.

16 Nell'analisi non sono stati inclusi il ms. Harley 4335-4339, latore della ritraduzione latina del volgarizzamento catalano, e il testimone castigliano D che reca un frammentario e anonimo Dialogo entre un sabio y una dueña, che segue in vari punti la traduzione della Consolatio nella redazione di Ginebreda, cfr. Pérez Rosado 1993: 113-120. 
permettono di ipotizzare una fascicolazione originaria (se si ammette una mancanza di sole $7 \mathrm{cc}$. carte nel primo fascicolo, senza supporre la presenza di un ulteriore fascicolo rilegato in testa al codice): I-III ${ }^{16}, \mathrm{IV}^{14}, \mathrm{~V}-\mathrm{VI}^{16}, \mathrm{VII}^{6}$, per un totale di 100 carte, di cui solo 76 si sono conservate. Le perdite $(24 \mathrm{cc}$. in totale) possono essere quantificate per ciascun fascicolo come segue: I (-11), II $(-7)$, III (-2), IV (-2), V (-1), VI (-1). Copiato da un'unica mano in scrittura gotica corsiva su una colonna di 19-21 linee; specchio di scrittura: 194x134 mm. Filigrana simile a Briquet 13262 («Roue du supplice, dite de Ste-Catherine», Francia del Sud, 1412-1421).

Iniziali dipinte in rosso o in blu all'inizio di ogni sezione di testo (rimangono visibili in molti casi le letterine guida), di cui 13 filigranate (alle cc. XIv, XxIVr, XXXVr, XXXXVIV, XXXVIIIV, XLr, XLVr, LIIIr, LXVIIr, LXXIXr, XCV, XCVr, XCVIIIV); iniziali maiuscole toccate in rosso all'interno del testo; spazi bianchi per le rubriche, che non sono state trascritte. All'angolo superiore destro di alcune carte è visibile un'antica numerazione in cifre romane in inchiostro rosso che termina a c. XCIx (il che, se si ammette l'ipotesi di fascicolazione proposta supra, porta a ipotizzare una prima carta non numerata). Lungo i margini sono presenti alcune note apposte dalla stessa mano che copia il testo e alcune prove di penna di mano differente e di epoca posteriore.

In corrispondenza dell'inizio di alcune sezioni di testo, nei margini accanto ai capolettera dipinti, è ancora visibile una numerazione in cifre romane toccate in rosso di mano coeva. Si tratta presumibilmente dell'indicazione di una ripartizione in capitoli - 35 in tutto - che non rispecchia la suddivisione della Consolatio in libri, metri e prose, e che risulta simile, ma non perfettamente sovrapponibile, a quella adottata anche nei codici R e N (cfr. infra \$2.3).

Il manoscritto, che si trovava in cattivo stato di conservazione, è stato nei mesi scorsi sottoposto a restauro e dotato di legatura; le carte sono state riordinate seguendo una numerazione moderna in cifre romane vergata a lapis posta all'angolo superiore di ciascun foglio, che riprende quella antica in inchiostro rosso. Durante il recente lavoro di restauro sono stati inseriti dei fogli bianchi in corrispondenza di quelli andati perduti.

\subsection{Contenuto}

Si fornisce di séguito un prospetto delle parti di testo conservate in J, indicando l'incipit e l'explicit di ciascuna sezione continua, e i passi del De consolatione $(C P h)^{17}$ a cui corrispondono. Si segue l'antica numerazione delle carte in cifre romane; le carte indicate come bianche sono quelle moderne inserite durante l'operazione di restauro del codice per dar conto dei fogli perduti. Si segnala, in aggiunta, la caduta di una foglio tra la c. XXviII e la c. XxIx, non indicata mediante inserzione di una pagina bianca.

17 Qui e più avanti si fa riferimento all'edizione della Consolatio curata da Bieler (1984). 
Chiara Concina. Primi sondaggi sul testimone J del volgarizzamento catalano della Consolatio Philosophiae

\begin{tabular}{|c|c|c|}
\hline cc. & Libro I & \\
\hline \multirow{2}{*}{ VIII-XI } & $\begin{array}{l}\text { c. VIIIr, inc: deuets notar que la philosofia tench manera de bon metge car axi com } \\
\text { lo metge demane } e \text { vol saber }\end{array}$ & CPb $1,2,1$ \\
\hline & c. XIv, expl.: E dix sens aço que yo te dit est axi com a ase qui on esturments & $C P h 1,4,1$ \\
\hline XII-XIV & bianche & \\
\hline \multirow[b]{2}{*}{ XV-XVIII } & $\begin{array}{l}\text { c. XVr, inc: pus cruelment punir que ara Empero si fos acusat de les dites coses } \\
\text { o semblants }\end{array}$ & $C P h 1,4,36$ \\
\hline & $\begin{array}{l}\text { c. XVIIIV, expl:: e conech en quina manera poras guarir car per ço com tes } \\
\text { exoblidada la sciencia }\end{array}$ & $C P h 1,6,17$ \\
\hline XIX-XXI & \multicolumn{2}{|l|}{ bianche } \\
\hline
\end{tabular}

\begin{tabular}{|c|c|c|}
\hline cc. & Libro II & \\
\hline \multirow{2}{*}{ XXII } & c. XXIIr, inc: dret Ara vull retornar deuers mi les mies mans quet hauia esteses & CPh II, 2, 5 \\
\hline & c. XXIIv, expl:: Encare mes not deu esser oblidat com paulus e & $C P h$ II $, 2,12$ \\
\hline XXIII & ( & \\
\hline \multirow{2}{*}{ XXIV } & c. XxIVr, inc:: a la hora nona es alt car no ha empatxament & CPh II, III, 1 \\
\hline & c. XXIVV, expl:: E com sia tan bona $e$ tan benigna $e$ vertadera & CPb II, 4, 6 \\
\hline XXV-XXVII & bianche & \\
\hline \multirow[b]{2}{*}{ XXVIII } & c. XXVIIIr, inc:: no ha prouat axi com aquel qui ho posseeix & CPh II, 4, 15 \\
\hline & c. XXVIIIV, expl:: Encare mes aquell qui ha abundancia dels bens de fortuna & $\begin{array}{l}C P h . \text { II, 4, } \\
25\end{array}$ \\
\hline \multirow[b]{2}{*}{ XXIX-XXXI } & c. XXIXr, inc:: ques mes les vols per seruir del cors & CPh II, 5, 15 \\
\hline & c. xxxiv, expl:: $e$ sobres de marauella excellencia que tant desigats les & CPh II, 6, 4 \\
\hline XXXII & bianca & \\
\hline \multirow[b]{2}{*}{ XXXIII-XXXVII } & c. XXXIIIr, inc:: presons En apres ell fo vençut e pres $e$ ach a soferir cruel preso & CPh II, 6,11 \\
\hline & $\begin{array}{l}\text { c. XXXVIIr, expl:: E uiuen mes bestialment que humanal per defelliment de vera } \\
\text { amor }\end{array}$ & CPh II, VIII \\
\hline
\end{tabular}

\begin{tabular}{|c|c|c|}
\hline $\mathrm{cc}$. & Libro III & \\
\hline \multirow[b]{2}{*}{ XXXVII-XLVI } & c. XXXVIIV, inc:: Quant la philosophia ach callat $e$ dites & CPh III, 1,1 \\
\hline & c. XLVIV, expl:: segueix se que los dits plaers corporals no puxe $n$ donar sobira be $\mathrm{E}$ & CPh III, 7,5 \\
\hline XLVII & bianca & \\
\hline \multirow[b]{2}{*}{ XLVIII } & c. XLVIIIr, inc:: sauietat En cascu $n$ trobaras manifestament freuoltat & CPh III, 7 \\
\hline & $\begin{array}{l}\text { c. XLVIIIV, expl:: no les serquats dins les aygues ni los peyes en los boscatges } \\
\text { Empero totes }\end{array}$ & $\begin{array}{l}\text { CPh III, VIII, } \\
6\end{array}$ \\
\hline
\end{tabular}


Chiara Concina. Primi sondaggi sul testimone J del volgarizzamento catalano della Consolatio Philosophiae

\begin{tabular}{|c|c|c|}
\hline XLIX-L & bianche & \\
\hline \multirow[b]{2}{*}{ LI-LXVII } & c. LIr, inc:: aquestes coses mortals $e$ transitories & CPh III, 9, 29 \\
\hline & $\begin{array}{l}\text { c. LXVIIr, expl:: e la claradat de la dita font no la pot cobrar si la pert a la fi de la nit } \\
\text { co es en la ora de la mort }\end{array}$ & $\begin{array}{l}\text { CPh III, XII, } \\
58\end{array}$ \\
\hline
\end{tabular}

\begin{tabular}{|c|c|c|}
\hline cc. & \multirow{2}{*}{\multicolumn{2}{|c|}{\begin{tabular}{l|l}
\multicolumn{2}{|c|}{ Libro IV } \\
c. LXVIIr, inc: \\
Quant la philosophia ach consolat boeci remouent aquelles coses & CPh IV, 1,1
\end{tabular}}} \\
\hline \multirow{3}{*}{ LXVII-LXXIV } & & \\
\hline & & \\
\hline & c. LXXIVv, expl.: los mals homens qui more $n$ en lur maluestat & $\begin{array}{l}\text { CPh IV, 4, } \\
22\end{array}$ \\
\hline \multirow[b]{3}{*}{ LXXVI-LXXVII } & \multicolumn{2}{|l|}{ bianca } \\
\hline & $\begin{array}{l}\text { c. LXXVIr, inc: benaunyrança E si per ventura pren la carrera comuna de les gents } \\
\text { que no han cura }\end{array}$ & $\begin{array}{l}\text { CPh IV, 28- } \\
29\end{array}$ \\
\hline & c. LXXVIIV, expl:: E la rao per quem marauell es aquesta com sapia que deus qui es & $\mathrm{CPh} \mathrm{IV}, 5,5$ \\
\hline LXXVIII & \multicolumn{2}{|l|}{ bianca } \\
\hline \multirow[b]{2}{*}{ LXXIX-LXXXIX } & $\begin{array}{l}\text { c. LXXIXr, inc: nes raho per que lo glas se fon per que apar que ignora } n \text { cia de raho } \\
\text { fa hom marauellar }\end{array}$ & CPh IV, v, 15 \\
\hline & c. LXXXIXv, expl:: les quals si les vençets guanyades les coses celestials $e$ perpetuals & CPh IV, VII \\
\hline
\end{tabular}

\begin{tabular}{|c|l|c|}
\hline CC. & \multicolumn{1}{|c|}{ Libro V } & CPh V,1,1 \\
\hline \multirow{2}{*}{ LXXXIX-XCIX } & $\begin{array}{l}\text { C. } \text { XXXXIXv, expl.: la sciencia de nostre senyor al qual res no pot esser amagat al qual } \\
\text { sia dada honor } e \text { lahor e gloria per tostemps in secula seculorum amen ffinito libro } \\
\text { sit laus gloria e christo amen }\end{array}$ & $C P, 6,48$ \\
\hline
\end{tabular}

\subsection{Partitura interna}

J presenta una segmentazione del testo che, come accade anche in $\mathrm{R} \mathrm{e} \mathrm{N}$, non conserva l'originaria distinzione in libri, metri e prose della Consolatio, che si ritrova invece nei manoscritti della redazione $\beta$ e nelle stampe. Pur avvicinandosi per assetto interno a quanto si rinviene in $\mathrm{R}$ e $\mathrm{N}$, i 35 capitoli in cui è ripartito $\mathrm{J}$ (che accorpano al loro interno e senza un'apparente giustificazione logica metri e prose del testo di Boezio), non seguono sempre l'articolazione proposta nei due manoscritti della redazione $\alpha$. La numerazione dei capitoli, a differenza di quanto si ha in $\mathrm{N}$ (la sezione tràdita da $\mathrm{R}$ risulta troppo esile per istituire un paragone attendibile, ma coincide sostanzialmente con quanto reperibile in $\mathrm{N}$ ), è continua, non riprende cioè dall'inizio in corrispondenza dell'apertura di ciascun libro, come invece accade per $\mathrm{N}$ in cui ogni libro è suddiviso come segue: $\mathrm{I}=13$ cap., II = 8 cap., III = 13 cap., IV = 7 cap., V = 7 cap. (Ziino 1997, 1: 57). R e N accorpano in genere in ogni capitolo 
un metro e una prosa, mentre $\mathrm{J}$ manifesta una tendenza a fondere anche più metri e prose di séguito $\mathrm{o}$, al contrario, a dedicare un capitolo a un solo metro o prosa.

Lo schema di sintesi che si propone infra tenta di dare conto della ripartizione testuale attuata in $\mathrm{J}$, ricostruibile in alcuni punti solo per via congetturale a causa delle perdite subíte dal codice e dal fatto che la numerazione dei capitoli posta a margine non è sempre leggibile. I numeri di capitolo posti tra parentesi indicano che la ricostruzione è dubbia. Nelle ultime due colonne di destra sono invece indicati i capitoli corrispondenti di $\mathrm{N}$ e di $\mathrm{R}$, che in alcuni casi concordano con quelli di J. Si ricorda che in $\mathrm{N}$ mancano le rubriche, ma che la suddivisione è restituita dalla tabula iniziale, mentre in $\mathrm{R}$ i numeri dei capitoli sono indicati in coda a ciascuna sezione testuale, prima degli spazi lasciati in bianco destinati ad accogliere le rubriche.

\begin{tabular}{|c|c|c|c|c|}
\hline Libro & Cap. & $\mathrm{CPh}$ & $\mathbf{N}$ & $\mathbf{R}$ \\
\hline \multirow{6}{*}{$\mathbf{I}$} & $(1)$ & (prologo?) / m.1/pr.1 & $\mathrm{I}-\mathrm{V}(1-15)$ & $\mathrm{I}-\mathrm{V}(1-13)$ \\
\hline & (2) & m.2/pr.2/m.3 & V (16-21)-VII & V (14-22)-VII \\
\hline & 3 & pr.3/m.4 & $\mathrm{VUL}$ & VU \\
\hline & 4 & pr.4/m.5 & IX-XI & \\
\hline & 5 & pr.5/m.6 & XII & \\
\hline & (6) & pr.6/m.7 & XIII & \\
\hline \multirow{8}{*}{ II } & (7) & pr.1/m.1 & $\mathrm{I}$ & \\
\hline & $(8)$ & pr.2/m.2 & II & \\
\hline & (9) & pr.3/m.3 & III & \\
\hline & $(10)$ & pr.4/m.4 & IV & \\
\hline & $(11)$ & pr.5/m.5/pr.6/m.6/pr.7 & V-VII $(1-23)$ & \\
\hline & 12 & m.7 & VII $(24-41)$ & \\
\hline & 13 & pr.8 & VIII $(1-14)$ & \\
\hline & 14 & m.8 & VIII $(15-21)$ & \\
\hline \multirow{12}{*}{ III } & 15 & pr.1/m.1 & $\mathrm{I}$ & \\
\hline & 16 & pr.2 & II $(1-25)$ & \\
\hline & 17 & m.2 & II $(26-36)$ & \\
\hline & 18 & pr.3/m.3/pr.4/m.4 & III-IV & \\
\hline & 19 & pr.5/m.5 & $\mathrm{V}$ & \\
\hline & 20 & pr.6/m.6 & VI & \\
\hline & (21) & pr.7/m.7 & VII & \\
\hline & $(22)$ & pr. $8 / \mathrm{m} .8$ & VIII & \\
\hline & $(23)$ & pr.9/m.9 & IX-X & \\
\hline & 24 & pr.10/m.10 & $\mathrm{XI}$ & \\
\hline & 25 & pr.11/m.11 & $\mathrm{XII}$ & \\
\hline & 26 & pr. $12 / \mathrm{m} .12$ & XIII & \\
\hline \multirow{3}{*}{ IV } & 27 & pr.1/m.1 & $\mathrm{I}$ & \\
\hline & 28 & pr.2/m.2/pr.3/m.3/pr.4/m.4/pr.5/m.5 & II-V & \\
\hline & 29 & pr.6/m.6/pr.7/m.7 & VI-VII & \\
\hline \multirow{4}{*}{$\mathbf{V}$} & 30 & pr.1/m.1 & $\mathrm{I}$ & \\
\hline & 31 & pr.2/m.2 & II & \\
\hline & 32 & $\begin{array}{l}\text { pr. } 3 / \text { pr. } 4 \text { (parz.) / pr.5 (par.) } / \mathrm{m.5} \\
\text { mancano i m. } 3 \text { e } 4\end{array}$ & III-V & \\
\hline & $33-35$ & pr. 6 & VI-VII & \\
\hline
\end{tabular}

3. Libri I-IV: lezioni caratteristiche di J, R, N, Se ${ }_{1}$ rispetto a $\beta$

Riprendendo lo spoglio dei loci che evidenziano i punti di accordo e di divergenza riscontrabili nel raffronto tra $\alpha$ e $\beta$ forniti nell'accurato lavoro di Francesca Ziino (1997, 1: 59-155), si propone 
qui una selezione di esempi dai quali emerge una chiara affinità testuale (lezioni caratteristiche, assenza/presenza di alcuni passi, etc.) tra $\mathrm{J}, \mathrm{R}, \mathrm{N}, \mathrm{Se}_{1}$ da un lato, che differisce spesso in modo evidente da quanto si legge nei testimoni della redazione $\beta .{ }^{18}$ Lo spoglio è volto a individuare la variante redazionale costituita da J e - sebbene coinvolga anche la segnalazione di una serie di errori monogenetici che investono la tradizione - esula per il momento da una discussione stemmatica stricto sensu.

I. $C P h$ I, III

$\mathrm{J}, \mathrm{R}, \mathrm{N}, \mathrm{Se}_{1}$ recano una versione molto ridotta e decisamente poco fedele del terzo metro del Libro $\mathrm{I}$, laddove $\beta$ offre invece una redazione più ampia e più aderente ai versi latini di Boezio. $\mathrm{J}, \mathrm{R} \mathrm{e}$ $\mathrm{N}$ concordano nel saldare il $\mathrm{m}$. III alla prosa che precede, mentre $\mathrm{Se}_{1}$ fonde il testo del $\mathrm{m}$. III con la pr. 2 e inserisce erroneamente dopo la rubrica una sezione desunta dalla pr. 3 che segue. Tale fraintendimento è spiegabile se si ipotizza per $\mathrm{Se}_{1}$ un modello affine a J, R o N, che non presentano un ripartizione precisa di metri e prose.

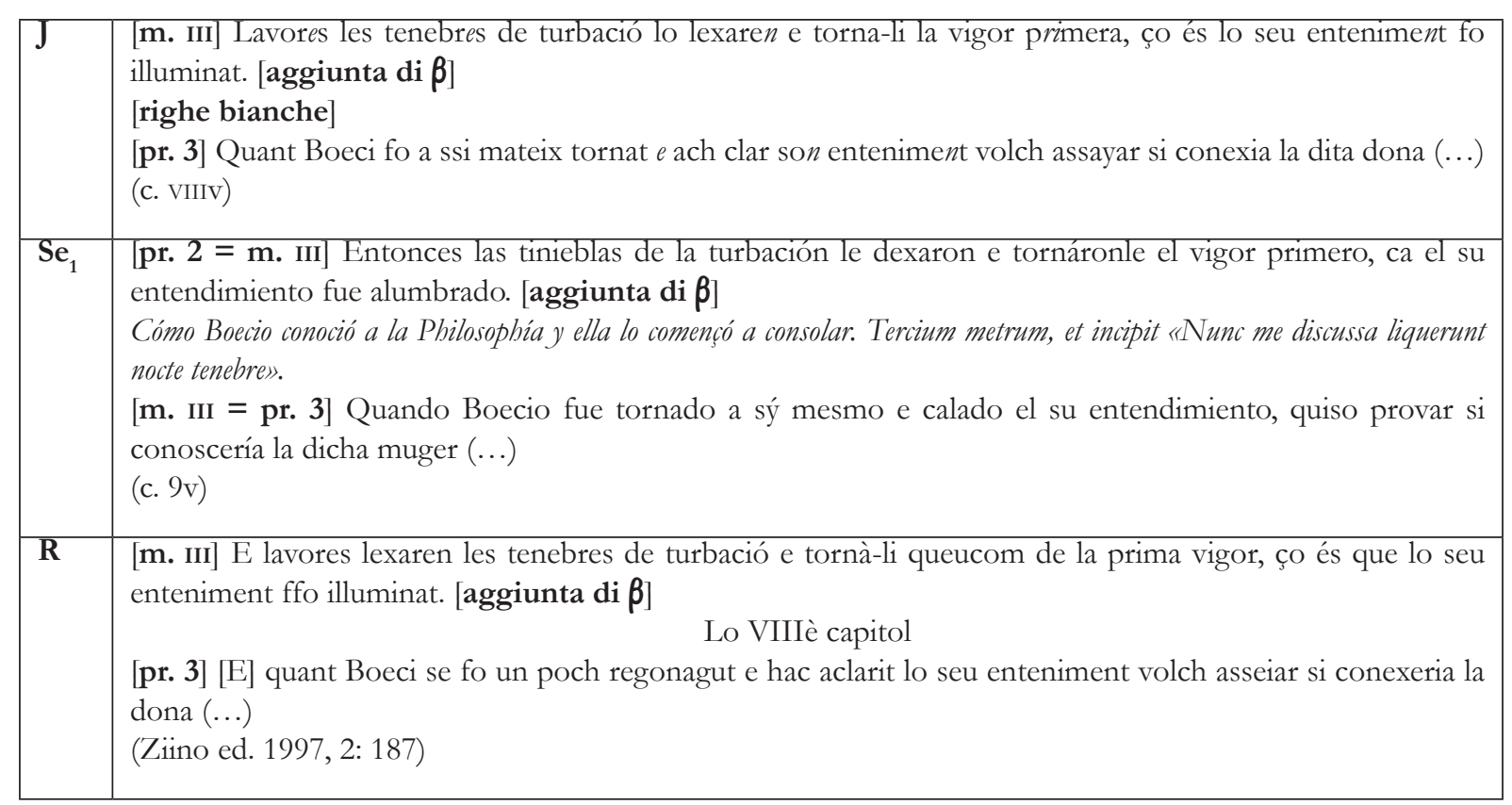

18 I passi di R e N sono tratti da Ziino (ed.) 1997, 2: 1-151 (N) e 178-191 (R); quelli di Se dall'ed. online curata Antonio Doñas per il numero speciale di Memorabilia: Boletin de Literatura Sapiencial Medieval, 8, 2004-2005: http://parnaseo.uv.es/ Memorabilia/memorabilia8/boecio/index.htm. Il testo di $\beta$ (per cui ci si basa sul ms. H = BSB, Cod. Hisp. 145) e di J viene fornito in trascrizione diplomatica, segnalando in corsivo lo scioglimento delle abbreviazioni. Le parentesi quadre indicano le integrazioni, le parentesi uncinate le espunzioni, $<\ldots>$ indica una lacuna e $(\ldots)$ una passo che non è stato trascritto per motivi di spazio. 


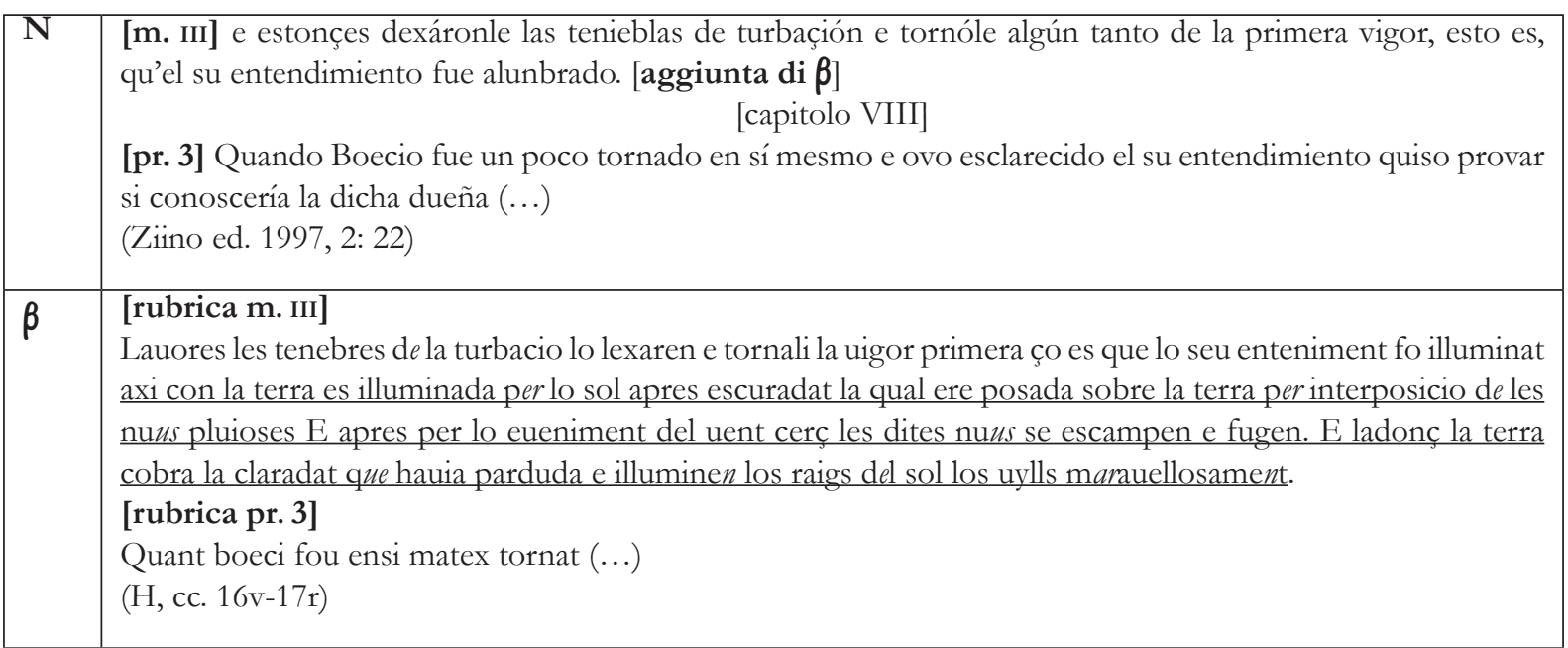

\section{II. $C P h \mathrm{I}, \mathrm{v}, 1-9$}

J concorda con $\mathrm{N} \mathrm{e} \mathrm{Se}_{1}$ nell'assenza di un passo presente invece in $\beta$. La lezione di questi tre testimoni risulta nel complesso errata rispetto a quanto si legge nei versi del De Consolatione, nei quali si dice che la luna è tanto più luminosa, e oscura dunque le altre stelle col suo chiarore, quanto più è distante dal sole. ${ }^{19} \mathrm{Il}$ senso corretto viene restituito in questo caso dall'integrazione presente in $\beta$.

\begin{tabular}{|c|l|}
\hline $\mathbf{J}$ & $\begin{array}{l}\text { O senyor Déu, creador dels çels } e \text { de totes coses } e \text { de totes les estelles, qui... forçes les estelles que.s moue } n \\
\text { aregladament, axí que la luna [aggiunta di } \beta \text { ] per plena que sia no amague les estelles, encare mes as ordonat } \\
\text { que la planeta que es apellada venus (...) } \\
\text { (c. xvIr) }\end{array}$ \\
\hline $\mathbf{S e}_{1}$ & $\begin{array}{l}\text { Oh, Señor Dios Criador de los cielos e de todas las cosas e de todas las estrellas, que... fazes a las estrellas que } \\
\text { se muevan regladamente, assý que la Luna, [aggiunta di } \beta \text { ] por llena que sea, no asconde las estrellas, e has } \\
\text { ordenado que la planeta que es llamada Venus (...) } \\
\text { (c. 13v) }\end{array}$ \\
\hline $\mathbf{N}$ & $\begin{array}{l}\text { O señor Dios, criador de los çielos e de todas las estrellas, el qual... mueves los çielos muy presta mente, bien } \\
\text { ansí que aunque la luna [aggiunta di } \beta \text { ] por llena que sea no encubre las estrellas; e aun más, as ordenado la } \\
\text { planeta que es llamada Venus }(. . .) \\
\text { (Ziino ed. 1997, 2: 34) }\end{array}$ \\
\hline
\end{tabular}

19 «O stelliferi conditor orbis, | qui perpetuo nixus solio | rapido caelum turbine uersas | legemque pati sidera cogis, | ut nunc pleno lucida cornu | totis fratris obuia flammis | condat stellas luna minores, | nunc obscura pallida cornu | Phoebo proprior lumina perdat», Bieler (ed.) 1984: 11. 
Chiara Concina. Primi sondaggi sul testimone J del volgarizzamento catalano della Consolatio Philosophiae

$\beta \quad O$ senyor deus creador dels cels e de totes coses e de totes les esteles qui... forçes les esteles que.s moguen regladament e que la luna com es molt luyn del sol sia plena e que tolga enuers lo nostra esgardament la claradat a les esteles qui son prop de ella e que com pus acostada es al sol que reebe enues lo mon maior (maior) claredat dell. Encara mes as ordonat que la planeta que es appellada Venus (...) (H, c. 26v)

III. $C P h \mathrm{I}, 5,5$

J, $\mathrm{Ne} \mathrm{Se}_{1}$ concordano nell'assenza della glossa presente invece in $\beta$.

\begin{tabular}{|c|c|}
\hline $\mathrm{J}$ & $\begin{array}{l}\text { E no saps en la leny molt antiga de la tua ciutat }(\ldots) \text { car aquell que es dins los valls e dins los murs de la dita } \\
\text { ciutat no li cal tembre que puxa merexer exill mas aquells qui ixen fora de la dita ciutat } e \text { fora los valls tantost } \\
\text { perden lo priuilegi [aggiunta di } \beta \text { ] Emperò yo més son estada somoguda }(. . .) \\
\text { (c. xVIIV) }\end{array}$ \\
\hline $\mathrm{Se}_{1}$ & $\begin{array}{l}\text { ¿E non sabes tú la ley muy antigua de la cibdad (...) ca aquellos que son dentro en los valles e muros de la } \\
\text { dicha cibdad non les cale temer que puedan merescer exulamiento, mas aquéllos que salen de la dicha cibdad } \\
\text { e fuera de los valles tan toste pierden el dicho privilegio. [aggiunta di } \beta \text { ] Empero yo me só sometida (...) } \\
\text { (c. 14r) }\end{array}$ \\
\hline $\mathbf{N}$ & $\begin{array}{l}\text { E no sabes tú la ley muy antigua de la tu cibdad (...) Ca aquél que es de dentro de los valles e de dentro de los } \\
\text { muros de la dicha çibdad no le cale temer que pueda meresçer destierro, mas aquéllos que salen de la dicha } \\
\text { cibdad e son fuera de los valles, conviene saber, o cavas, tantost pierden el dicho privillejo. [aggiunta di } \beta \text { ] } \\
\text { Enpero más só yo estada movida (...) } \\
\text { (Ziino ed. 1997, 2: 35-36) }\end{array}$ \\
\hline$\beta$ & $\begin{array}{l}\text { E no saps tu la ley entiga de la tua ciutat }(. . .) \text { car aquell qui es dins los uals e dins los murs de la dita ciutat } \\
\text { no li calra tenbre que meresque exill mas aquells qui ixen de la dita ciutat e fora los ualls tantost perden lo dit } \\
\text { priuelegi Aço uol dir la philosofia sots semblanca con per aquesta ciutat entén justícia de la qual negun no pot } \\
\text { ésser exillat si donç ell no la desempara la qual ciutat seguex déu. Emperò yo més són estada somoguda }(. . .) \\
(\mathrm{H}, \mathrm{c} .28 \mathrm{r} / \mathrm{v})\end{array}$ \\
\hline
\end{tabular}

Iv. $C P h$ I, vI, 5-6

Una situazione affine a quelle fino ad ora elencate si presenta anche per il $\mathrm{m}$. vI del Libro $\mathrm{I}$, in cui $\mathrm{J}, \mathrm{N}$ e Se${ }_{1}$ concordano nell'assenza di una precisazione presente invece in $\beta$.

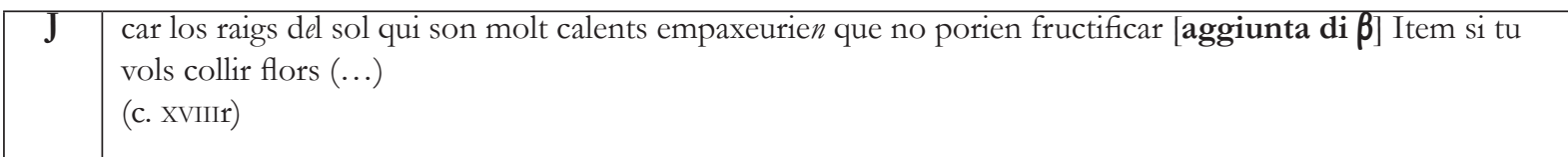




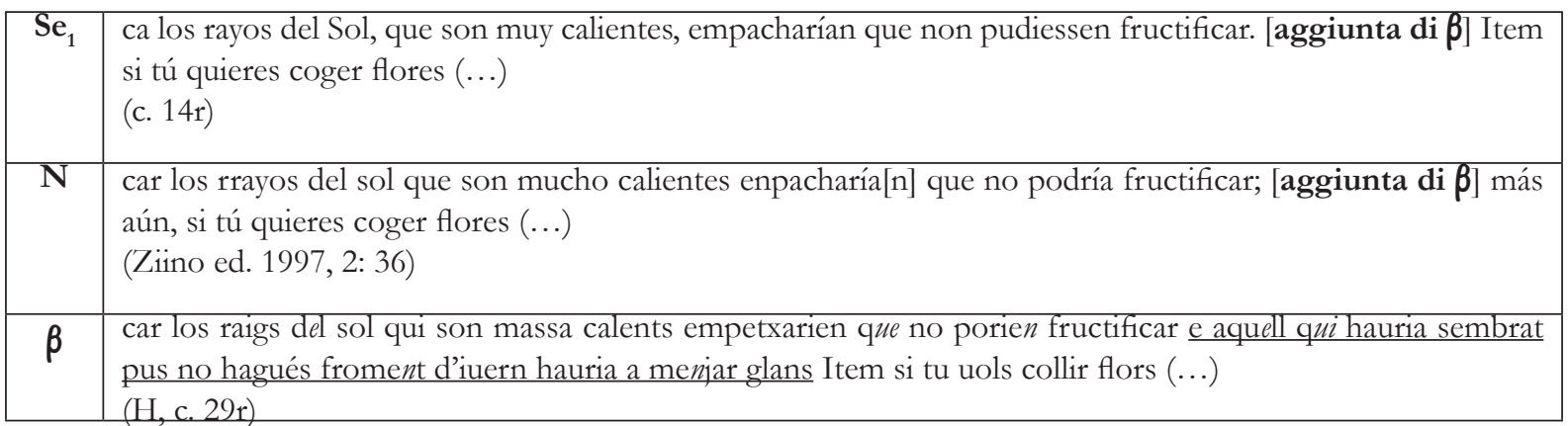

\section{v. $C P h$ II, 2,11}

J concorda con $\mathrm{N}$ e $\mathrm{Se}_{1}$ contro $\beta$, che inserisce una glossa tratta dal commento latino di Nicholas Trevet (cfr. Ziino ed. 1997, 1: 98).

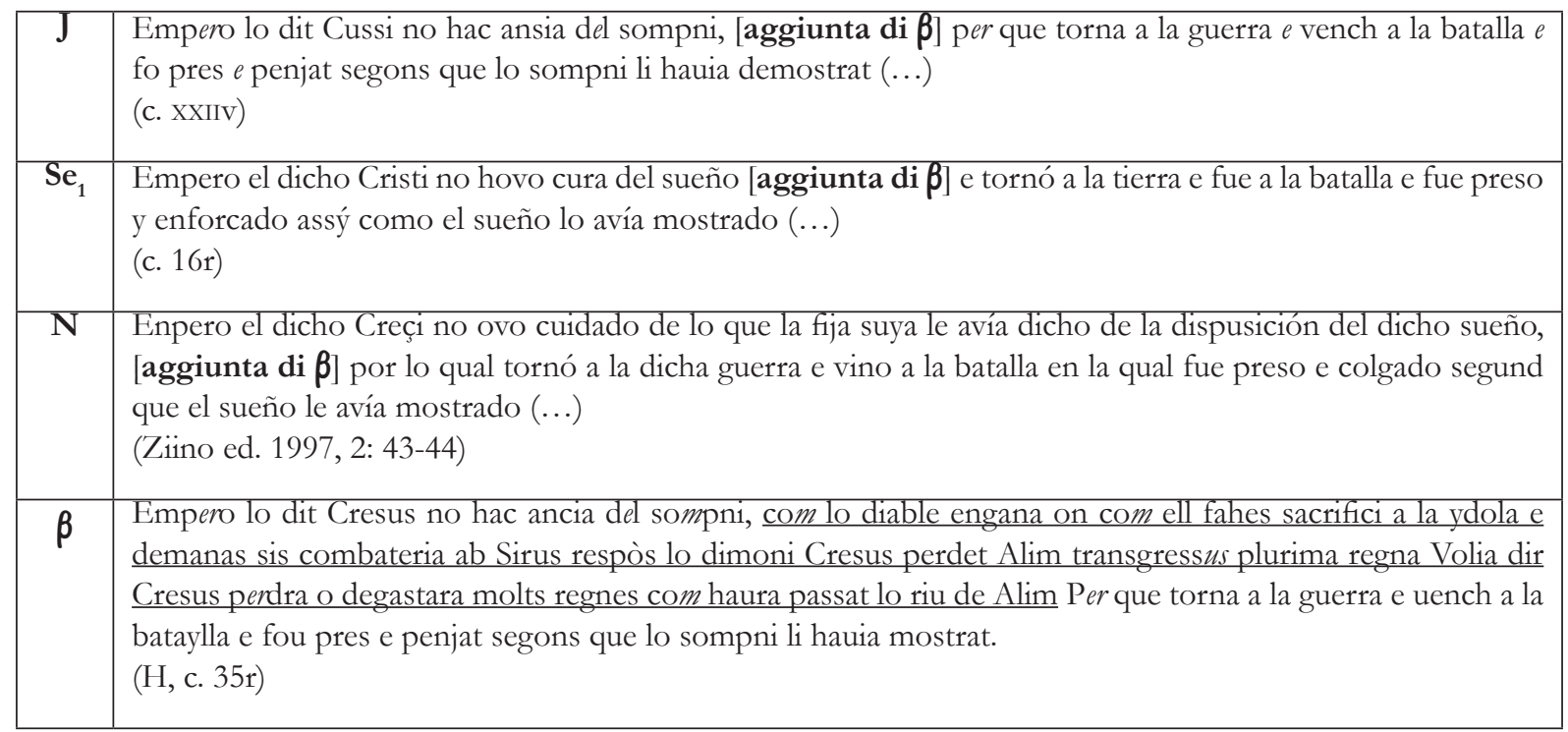

\section{vi. $C P h$ III, 5,8}

Nel brano in esame si riscontra l'accordo pieno tra $\mathrm{J}$ e $\mathrm{Se}_{1}$; $\mathrm{N}$ non risulta invece perfettamente sovrapponibile poiché in esso si conserva il sintagma che precede il passo aggiunto in $\beta$ (che ha l'assetto di una glossa didattico-moraleggiante introdotta da «Donques iutgeras»). Si noti inoltre che l'omissione presente in $\mathrm{J}$ e $\mathrm{Se}_{1}$ è probabilmente dovuta a una lacuna per saut du même au même da regne al secondo rregno, che si legge anora alla fine della frase conservata in $\mathrm{N}$. 


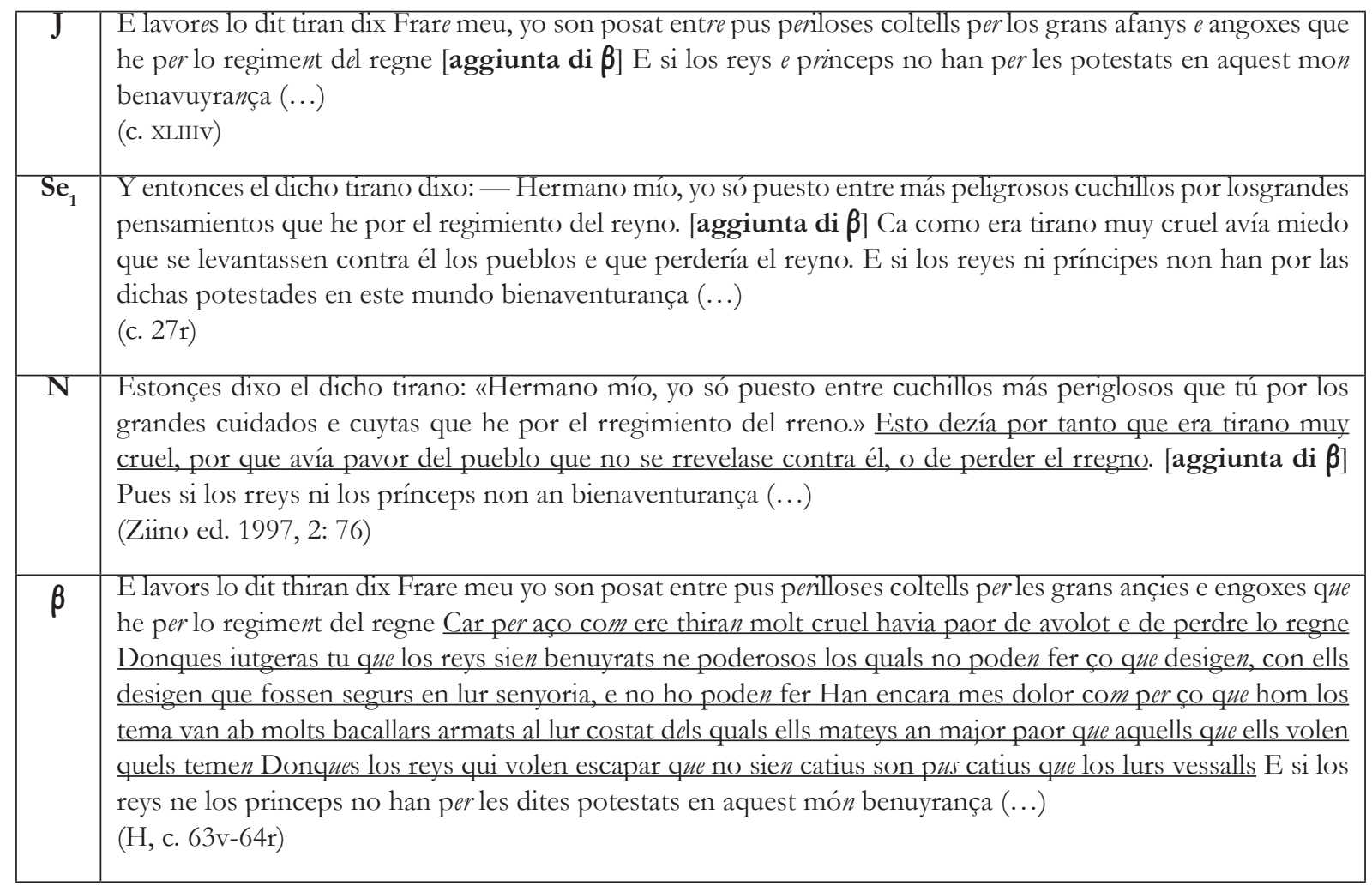

\section{viI. $C P h$ III, 8,10}

Anche nel celebre passo dell'ottava prosa del terzo libro, in cui Boezio fa riferimento al «pulcherrimum corpus» (Bieler ed. 1984: 48) di Alcibiade, il testo di J, N, Se $e_{1}$ diverge da $\beta$, in cui è inserita una glossa che amplia il testo desunta forse dal commento di Trevet (Ziino ed. 1997, 1: 99). ${ }^{20} \mathrm{Si}$ ricorda che il fraintendimento che si ritrova nella glossa di Trevet, per cui Alcibiade viene

20 «QUOD SI UTERENTUR HOMINES LINCEIS OCULIS, UT AIT ARISTOTELES, UT EORUM VISUS OBSTANCIA scilicet corpora opaca PENETRARET, NONNE ILLUD CORPUS ALCIPIADIS SUPERFICIE PULCHERRIMUM INTROSPECTIS VISCERIBUS TURPISSIMUM VIDERETUR? quasi diceret palam est quod sic. Quod uisus lincis obstancia penetret nec ab Aristotile nec ab alico auctentico inueni. Ysidorus enim ubi supra loquens de lince dicit sic: linx dictus quod in luporum genere numeratur bestia maculis distincta tergo ut pardus sed similis lupo, unde et ille licos iste linx dicitur. Huius urina conuerti in duriciam preciosi lapidis dicunt qui ligurius appellatur, quod et ipsas linces sentire hoc documento probatur. Nam egestum liquorem harenis in quantum poterunt contegunt inuidia quadam nature ne talis egestacio transeat in usum humanum. Linces dicit Plinius Secundus, extra unum non admittere 〈fetum〉. Vel autem dicit hic Ysidorus de penetracione uisus quamuis celebre sit quod uisu corpora densa penetrat. Dicit autem commentator hic quod Alcibiades meretrix quedam pulcherrima erat quem uidentes discipuli quidam Aristotelis ut eam uideret ad illum eam adduxerunt. Qui uisa ea dixit: si homines linceos oculos haberent ut obstancia queque penetrarent introspectis uisceribus corpus quod apparet pulcherrimum 
scambiato per una donna, ha origini antiche che risalgono almeno all'epoca carolingia, come si legge nel commento alla Consolatio di Rémi d'Auxerre (Courcelle 1967: 258). Tale confusione passa poi in molti volgarizzamenti, come ad esempio quelli antico-francesi (Langlois 1896 e Babbi 2010: 61-63).

\begin{tabular}{|c|l|l|}
\hline $\mathrm{J}$ & $\begin{array}{l}\text { E per aço Aristotil dix una vegada a alguns que mirauen una bella fembra } e \text { parlauen daquella si us pensavets } \\
\text { ço que es dins ella amagat [aggiunta di } \beta \text { ] ço queus es verares que sia bel conexeriets que es molt sutze car } \\
\text { sutzura qui es molt gran es amagada sots aquella color que veets (...) } \\
\text { (c. xLVIIIr) }\end{array}$ \\
\hline $\mathbf{S e}_{1}$ & $\begin{array}{l}\text { E por esto dixo Aristotiles una vegada a unos que miravan a una muger muy fermosa e fablavan d'ella: Si } \\
\text { pensades lo que es en ella dentro ascondido, [aggiunta di } \beta \text { ] lo que vos parece fermoso conosceríades que } \\
\text { es muy suzio, ca muy grand suziedad es ascondida de yuso de aquella color que vedes (...) } \\
\text { (c. 29r) }\end{array}$ \\
\hline $\mathbf{N}$ & $\begin{array}{l}\text { E per esto dixo Aristo[ti]les una vegada ha algunos que myravan una fenbra e fablavan de la su belleza: } \\
\text { «Si vos pensáis en aquello que en ella está ascondido, [aggiunta di } \beta \text { ] aquello que vos semeja que sea muy } \\
\text { fermoso conosçeries que es muy suzio; car gran suziedat está escondida so aquella color que veedes (...) } \\
\text { (Ziino ed. 1997, 2: 81-82) }\end{array}$ \\
\hline$\beta$ & $\begin{array}{l}\text { E per aço Aristotil dix una vegada a alscuns qui miraven una bella fembre qui ere avol de son cors appellada } \\
\text { Alcipies e parlaven d'aquella: Si us pensavets en ço que es dins en ella amagat en lo cors de Alcipues e ho } \\
\text { podiets veire ab uylls de linx aço que appar que sia bell apparria fort leig Linx es una bestia axi gran com un } \\
\text { molto la qual ha tan gran agudesa de vista que paret ne rocha no li empatxa e ha la orina tan virtuosa que } \\
\text { ella tota hora que deu orinar caua en terra per tal que no sia trobada Empero de la sua orina se fa una pedre } \\
\text { preciosa que es appellada ligurius la qual bestia es en India Mas açò (...) } \\
\text { (H, c. 69v) }\end{array}$ \\
\hline
\end{tabular}

vIII. CPh III, 11, 37-41

$\mathrm{J}, \mathrm{N}$ e $\mathrm{Se}_{1}$ non contengono l'ultima parte della prosa 11 del terzo libro, mentre $\beta$ «traduce più esattamente i paragrafi 37-41 della prosa» (Ziino ed. 1997, 1: 104-105). La già rilevata errata ripartizione fra pr. $11 \mathrm{e} \mathrm{m}$. XII di Se ${ }_{1}$ potrebbe essere spiegata anche qui da un'assenza di demarcatori posti a scandire la partitura interna tra metri e prose presente nel modello di questo incunabolo.

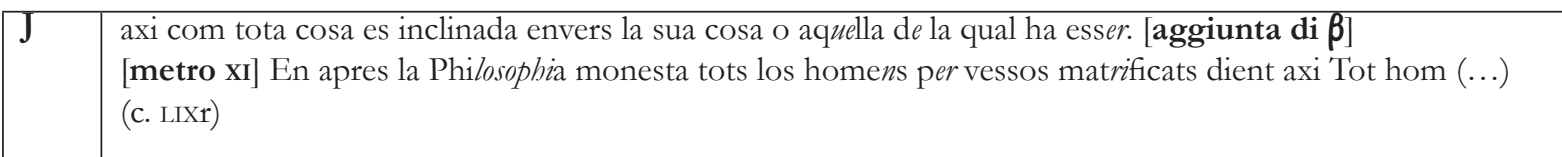

turpissimum uideretur» (Silk. ed. online; ma cfr. anche Isidoro, Etym. XII, 2, 20). In Trevet è tuttavia assente il riferimento all'India come luogo di origine della lince, che si ritrova invece altrove, come per esempio nell'anonimo commento alla Consolatio (XII secolo), edito da E. Silk, che si rifà, a sua volta, a un’informazione desunta da Solino («Lynx dicitur extremus fetus lupi, sed Solinus refert in India plures esse lynces ubi nullus lupus est», Silk ed. 1935: 144; ma si veda anche l'accenno in Ovidio, Metam. XV, 413-415). 
Chiara Concina. Primi sondaggi sul testimone J del volgarizzamento catalano della Consolatio Philosophiae

\begin{tabular}{|c|c|}
\hline $\mathrm{Se}_{1}$ & $\begin{array}{l}\text { assí como toda cosa es ynclinada a la su causa o más a aquélla de la qual ha estado. [aggiunta di } \beta \text { ] } \\
\text { Después la Philosofía amonesta a todos los ombres por versos metrificados diziendo assí: } \\
\text { Undecima metrum, et incipit «Quisquis profunda mente». En aqueste metro dize que <cosa sonl> hombre puede por estudio } \\
\text { conoscer lo que ante ygnorava } \\
\text { Todo hombre (...) } \\
\text { (c. 33v) }\end{array}$ \\
\hline $\mathbf{N}$ & $\begin{array}{l}\text { así como toda cosa es ynclinada contra el su començamiento e fyn. [aggiunta di } \beta \text { ] } \\
\text { [metro xr] E pues la Philosofía amonesta a todas las gentes diziendo ansí: Todo omne (...) } \\
\text { (Ziino ed. 1997, 2: 95) }\end{array}$ \\
\hline$\beta$ & $\begin{array}{l}\text { axi com tota cosa es inclinada envers la sua causa e mes a aquella de la qual ha esser Mas ja havem mostrat } \\
\text { que esser un es esser be Dix Boeci ver es Donchs dix la Philosophia totes coses desigen be la deffinicio del } \\
\text { qual es aquesta (...) } \\
{[\text { metro xi] En apres la Philosofia amonesta tots los homens per versos metrifichats dihent axi Tot hom (...) }} \\
(\text { H, c. } 82 \mathrm{r} / \mathrm{v})\end{array}$ \\
\hline
\end{tabular}

\section{Lezioni corrette di J, R, $\mathrm{N}, \mathrm{Se}_{1}$ contro errori presenti in $\beta$}

In alcuni casi $\mathrm{J}, \mathrm{Se}_{1}$, $\mathrm{R}$ e $\mathrm{N}$ concordano nel tramandare la lezione corretta contro un errore comune al resto della tradizione, per cui si riportano qui due casi a titolo di esempio.

\section{I. $C P h$ I, 3, 13-14}

Il modello diretto del passo in esame è costituito da una glossa del commento di Guglielmo d'Aragona: «Vnde omnia ista breuiter et optime significauit Empedocles, ut refert Theofrastus principio De plantis, dicens (...)» (Olmedilla Herrero ed. 1997: 42). Il fraintendimento planetes per plantes è in verità assai banale, tuttavia l'accordo compatto di tutti i testimoni di $\beta$ nel riportare l'errore contro la lezione corretta che si legge in $\mathrm{R}, \mathrm{N}, \mathrm{J}$ e $\mathrm{Se}_{1}$, lascia presagire un problema di lettura situato nel modello al quale farebbero capo i codici di $\beta$.

\begin{tabular}{|c|ll|}
\hline $\mathbf{R}$ & $\begin{array}{l}\text { segons que recomte Theofatus en el començamiento dell libre De plantes } \\
\text { c. } 145 \mathrm{v}\end{array}$ & $\begin{array}{l}\text { (Ziino ed. 1997, } \\
\text { 2: 190) }\end{array}$ \\
\hline $\mathbf{N}$ & $\begin{array}{l}\text { segund que rrecuenta Theofanus en el començamiento del libro De plantes } \\
\text { c. } 13 \mathrm{v}\end{array}$ & $\begin{array}{l}\text { (Ziino ed. 1997, } \\
2: 25)\end{array}$ \\
\hline $\mathbf{J}$ & $\begin{array}{l}\text { segons que compte Trophastus en lo començament del libre de plantes } \\
\text { (cc. Xv-XIr) }\end{array}$ & (c. 10v) \\
\hline $\mathbf{S e}_{1}$ & según cuenta Theofatícolo en el comienço del libro de plantes $(\ldots)$ & (...) \\
\hline
\end{tabular}


Chiara Concina. Primi sondaggi sul testimone J del volgarizzamento catalano della Consolatio Philosophiae

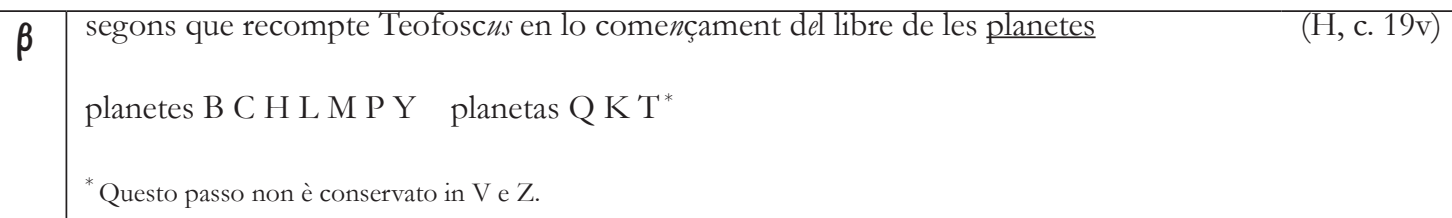

\section{II. $C P h$ III, 2, 1}

$\mathrm{J}, \mathrm{N}$ e Se ${ }_{1}$ tramandano la lezione corretta laddove $\mathrm{i}$ testimoni del gruppo $\beta$ presentano invece una cattiva lettura del testo latino della Consolatio: «Tum defixo paululum uisu et uelut in augustam suae mentis sedem recepta sic coepit» (Bieler ed. 1984: 38).

\begin{tabular}{|c|c|c|}
\hline $\mathrm{J}$ & tine $n$ t la vista fitada envers la terra e la pensa en alt començament & (c. XXXVIIIV) \\
\hline $\mathrm{Se}_{1}$ & teniendo la vista fincada contra la tierra y el pensamiento en alto començamiento & (c. 24r) \\
\hline $\mathbf{N}$ & teniendo la vista contra la tierra y el pensamiento en alto començamiento & $\begin{array}{l}\text { (Ziino ed. 1997, } \\
2.68)\end{array}$ \\
\hline$\beta$ & $\begin{array}{l}\text { tenent la uista ficada en terra e la pensa en alt alcu } n \text { couinent comencament } \\
\text { en alt algun couinent començament B K e algun c. L } \\
\text { e ella pença en algun mouiment } \mathrm{C} \mathrm{M} \mathrm{Y} \\
\text { en alt algun couinentment e fo comensament P } \\
\text { e ella penso en algund començamiento conuiniente Q T* } \\
\text { * Ancora una volta } \mathrm{V} \text { e } \mathrm{Z} \text { non conservano il passo. }\end{array}$ & $(\mathrm{H}, \mathrm{c} .57 \mathrm{r})$ \\
\hline
\end{tabular}

4. Passi di J e $\mathrm{Se}_{1}$ che concordano $\operatorname{con} \beta$ contro $\mathrm{N}$

Raramente le lezioni di J e $\mathrm{Se}_{1}$ concordano con $\beta$ contro N. Ciò si verifica sempre quando N presenta errori che gli sono propri (n. I) o lectiones singulares che possono essere considerate innovazioni di N contro il resto della tradizione (n. II). ${ }^{21}$

\section{I. $C P h$ IV, vII, 17}

Il modello di questo passo del settimo metro del quarto libro è il commento di Guglielmo d'Aragona

21 Ma si vedano anche i casi già segnalati in Ziino ed. 1997, 1: 101 in cui N presenta degli inserti in corrispondenza di CPh III, 12, 24 e di CPh IV, 3, 16-17, non presenti nel resto della tradizione. 
che glossa una delle fatiche di Ercole (il furto dei pomi delle Esperidi): «Hesperides enim dicuntur esse quatuor Athlantis regis Affrice filie» (Olmedilla Herrero ed. 1997: 300-301). Il fraintendimento (rey de Greçia per Africa) di N si presenta qui isolato contro il resto della tradizione:

\begin{tabular}{|l|l|l|}
\hline $\mathbf{N}$ & Atalante Rey de Greçia & (Ziino ed. 1997, 2: 129) \\
\hline $\mathbf{J}$ & Atalatan rey dafrica & c. LXXXVIIIv \\
\hline $\mathbf{S e}_{1}$ & Athalante rey de África & (c. 44v) \\
\hline $\boldsymbol{\beta}$ & Atalante rey de Affricha & H, c. 114v \\
\hline
\end{tabular}

\section{II. $C P h$ IV, VII}

Ancora una volta il testo traduce il commento di Guglielmo d'Aragona: «De orto istarum poma subtrahit sapiens quia per hec ad philosophiam ueniens bonis intelligibilibus ditatur sensibilia deuincens, que loco draconis habentun» (Olmedilla Herrero ed. 1997: 301). In questo punto J concorda $\operatorname{con} \beta$, dove invece $\mathrm{N}$ aggiunge una glossa che si presenta come un'innovazione che gli è propria rispetto al resto della tradizione. J presenta invece una svista leggendo donar al posto di donques (attestato in $\mathrm{Se}_{1}$ e $\beta$ ).

\begin{tabular}{|l|l|}
\hline $\mathbf{N}$ & $\begin{array}{l}\text { Quieren dezir que la persona sabia deve sacar del huerto o del vergel mui Rico e deleitoso, conviene saber } \\
\text { de las Escripturas que son ordenades a Dios, deve sacar las dichas quatro cosas e synse aquéstas no las avría }\end{array}$ \\
\hline $\mathbf{J}$ & $\begin{array}{l}\text { (Ziino ed. } 1997,2: 129) \\
\text { (c. } \text { LxxxviII) }\end{array}$ \\
\hline $\mathbf{S e}_{1}$ & $\begin{array}{l}\text { del huerto d'estos, pues deve traer los pomos de oro la persona sabia, que es sciencia } \\
\text { (c. } 44 \mathrm{v})\end{array}$ \\
\hline $\mathbf{\beta}$ & $\begin{array}{l}\text { del ort daquestes donques deu traure los poms daur la persona sauisa ço es sciencia } \\
\text { (H, c. } 114 \mathrm{v})\end{array}$ \\
\hline
\end{tabular}

\section{Il Libro V: un problema aperto}

Il quinto libro costituisce uno dei nodi più problematici e complessi della tradizione manoscritta del Boezio catalano. Il fulcro della questione, e delle ipotesi che ruotano intorno ad essa, è da individuarsi nelle affermazioni contenute nel prologo di Ginebreda, ${ }^{22}$ in cui il revisore-traduttore

22 Il prologo è conservato integralmente dal ms. catalano $\mathrm{K}$, nel codice castigliano Q e nelle stampe castigliane $\mathrm{Se}_{1}\left(\mathrm{Se}_{2}\right.$, 
dichiara:

a) di essere intervenuto su un testo originariamente dedicato all'infante Giacomo di Maiorca (e ciò coincide con quanto sappiamo dalla trascrizione del perduto prologo del ms. di Montserrat, in cui troviamo, in aggiunta, anche il nome di Pere Saplana): «algunos han fecho todo su poder de romançar el dicho libro a instrución de los que non saben sciencia, et entre los otros ay uno, el qual le dirige al infante de Mallorca» (Riera i Sans 1984: 309).

b) di aver sistemato i numerosi difetti presenti nel modello: «E por quanto en la dicha exposición avía algunos defectos, especialmente porque el dicho libro esponedor dexa del quinto libro la quarta e la cinquena prosas e el tercero et el quarto metros; et essomesmo por quanto en el comienço del dicho libro non era la storia de Theodorico nin la persecución de Boecio nin el título del dito libro, nin essomesmo en el dicho libro non oviesse tabla, et essomesmo dentro la obra oviesse muchas otras faltas» (Riera i Sans 1984: 309).

Ginebreda si sarebbe dunque preoccupato non solo di aggiungere il titolo, la tabula e di introdurre nella sezione iniziale dell'opera, il volgarizzamento della storia di Boezio e Teodorico desunta dal Trevet - sostituendola a quella tratta dal prologo di Gugliemo d'Aragona (che si legge invece in $\mathrm{R}$ e $\mathrm{N}$ ) -, ma soprattutto di aver integrato le traduzioni del terzo e del quarto metro e della quarta e quinta prosa del quinto libro che, stando a quanto afferma Ginebreda, mancavano nel volgarizzamento di Saplana.

Quest'ultimo punto costituisce una delle questioni irrisolte della vicenda testuale del volgarizzamento Saplana-Ginebreda della Consolatio, dal momento che è stato osservato che N, pur presentando per questi passi lezioni che divergono decisamente da $\beta$, tramanda integralmente le traduzioni dei brani del quinto libro menzionati da Ginebreda. Se Jaume Riera i Sans (1984: 316) supponeva che le sezioni in questione "no faltaven a la versió original», ma solo nel codice di cui si servì Ginebreda, per contro secondo González Rolan e Saquero Suárez-Somonte e Ziino (ed. 1997: 153154) il verbo 'dexar' usato da Ginebreda nel prologo «no quiere significar «omitir», sino «reducir», «abreviar» la parte del texto en cuestión» (González Rolan / Saquero Suárez-Somonte 1992: 333). Stando a questa ipotesi si sarebbe trattato dunque di un'operazione di integrazione e revisione di un testo che non si presentava lacunoso, ma solo troppo sintetico.

Va inoltre aggiunto che alcuni chiarimenti sulla questione vengono offerti dalle recenti disamine condotte sulla traduzione ebraica di Shemuel Benveniste. Tale volgarizzamento venne esemplato su un modello catalano che conteneva probabilmente la redazione di Saplana accompagnata, lungo i margini, dalle glosse e dalle aggiunte di Ginebreda. Proprio a questo riguardo Ziino (2010: 391) ha rilevato:

In that book [V], where in proses 4-5 and metres 3-5, the difference between Ginebreda

To) e T, per le varianti che si riscontrano nel prologo dei vari testimoni cfr. ancora Riera i Sans 1984. 


\title{
Chiara Concina. Primi sondaggi sul testimone J del volgarizzamento catalano della Consolatio Philosophiae
}

\begin{abstract}
ans Saplana is patent and each text develops in an almost totally different way, Benveniste almost always translates Saplana's version as we read it in the Madrid MS. As a matter of fact Benveniste shows not to ignore Ginebreda's version: in a case (prose $4^{\text {th }}$ ), he quotes a passage from Ginebreda, marking it as an insertion from a different text into his main source.
\end{abstract}

A ciò si aggiunga che Benveniste riporta in appendice alla sua traduzione il testo della prosa 4, del metro 4 e della prosa 5 del quinto libro desunti da Ginebreda, per cui Zonta ha proposto che per questo punto «la fonte letteraria impiegata da Shemuel Benveniste fosse proprio la redazione, andata poi perduta, della versione catalana del De consolatione redatta da Antoni Ginebreda» (Zonta 2010: 309). Un volgarizzamento dunque, quello ebraico, esemplato a partire da una fonte assai prossima alla redazione costituita dal testo di Saplana e recante in aggiunta anche gli interventi che Ginebreda ultimò verso il 1390.

Se si analizza il comportamento di J per quel che concerne il quinto libro, si noterà innanzitutto che, pur con qualche omissione per cui concorda con $\mathrm{Se}_{1}$ contro $\beta$, esso segue in linea di massima quanto si trova in $\beta$ e si allontana dalle lezioni tràdite da $\mathrm{N}$, che differiscono spesso in modo vistoso tanto da non essere sovrapponibili con il resto della tradizione (Ziino ed. 1997, 1: 143-155). La terza prosa di J corrisponde a quanto si legge in $\beta$, ma in esso mancano del tutto proprio due dei quattro passi citati da Ginebreda nel prologo, cioè quelli corrispondenti al terzo e quarto metro, mentre si conservano unicamente la sezione iniziale della quarta prosa e l'ultima parte della quinta (che risultano saldate insieme; cfr. Fig. 1). Per questi lacerti il testo coincide in maniera precisa con quello di $\beta$. Infine il quinto metro, che è copiato di séguito alla frase finale della quinta prosa, corrisponde ancora una volta a quello tramandato da $\beta$. Se confrontata con quella di $\beta$ e Se la situazione di J può essere schematizzata come segue:

\begin{tabular}{|c|c|c|}
\hline$B$ & $\mathrm{~J}$ & $\mathrm{Se}_{1}$ \\
\hline testo completo pr. 3 & testo completo pr. $3=\beta$ & parte del testo pr. $3=\beta$ \\
\hline testo completo m. III & manca m. III & $\begin{array}{l}\text { manca il m. III: al suo posto } \\
\text { viene interpolata la storia di Tiresia } \\
\text { desunta dalla pr. } 3\end{array}$ \\
\hline $\begin{array}{l}\text { testo completo pr. } 4 \\
(\ldots) \text { Respos la philosophia e dix } \\
\text { aquesta questio ia es determanada } \\
\text { no pas perfetame } n \text { t (...) la francha } \\
\text { uoluntat romandra entegrament en } \\
\text { sa uirtut Posem que no fos neguna } \\
\text { presciencia (...) } \\
(\mathrm{H}, \text { c. } 125 \mathrm{v})\end{array}$ & $\begin{array}{l}\text { parte iniziale pr. } 4 \text { : } \\
\text { Respos la philosofia aquesta questio } \\
\text { ja es estada determinada no pas } \\
\text { perfetame } n \text { t }(. . .) \text { la francha voluntat } \\
\text { derogant entegrament en sa virtut }\end{array}$ & $\begin{array}{l}\text { testo completo pr. } 4=\beta \\
(\ldots) \text { Respondió la Philosofía e dixo: } \\
\text { Aquesta qüestión ya es determinada, } \\
\text { mas no perfectamente }(. . .) \text { la franca } \\
\text { voluntad remedará enteramente en } \\
\text { su virtud. Pongamos que no fuesse } \\
\text { ninguna presciencia }(. . .) \\
\text { (c. } 45 \mathrm{v})\end{array}$ \\
\hline testo completo m. IV & manca m. IV & testo completo $\mathrm{m} . \mathrm{IV}=\beta$ \\
\hline
\end{tabular}




\begin{tabular}{|c|c|c|}
\hline $\begin{array}{l}\text { testo completo pr. } 5 \\
(\ldots) \text { en aço deus molt e souin } \\
\text { pensar en les condicions de deu que } \\
\text { sobrepuge a enteniment humanal e } \\
\text { seras en aço que a com semblant als } \\
\text { angels qui naturalment son inclinats } \\
\text { en pensar en les coses diuinals. } \\
(\mathrm{H}, \text { c. } 130 \mathrm{r})\end{array}$ & $\begin{array}{l}\text { frase finale pr. 5: } \\
\text { En aço deuets molt } e \text { souen pensar } \\
\text { ço es en les condicions de deu que } \\
\text { sobrepuien a enteniment humanal E } \\
\text { seras en aço que a com semblant als } \\
\text { angels qui naturalment son inclinats } \\
\text { en pensar en les coses diuinals } \\
\text { (c. XCIVr/v) }\end{array}$ & $\begin{array}{l}\text { testo completo pr. } 5=\beta \\
\text { (...) Y en esto deves muy a menudo } \\
\text { pensar, es a saber en las condiciones } \\
\text { de Dios, que sobrepujan el } \\
\text { entendimiento humanal, e serás } \\
\text { en esto assý como semblante a } \\
\text { los ángeles, que naturalmente son } \\
\text { inclinados en pensar en las cosas } \\
\text { divinales. } \\
\text { (c. } 48 \mathrm{v})\end{array}$ \\
\hline testo completo $\mathrm{m} . \mathrm{V}$ & testo completo m. $v=\beta$ & testo completo $\mathrm{m} . \mathrm{v}=\beta$ \\
\hline
\end{tabular}

Si noterà ancora una volta che $\mathrm{Se}_{1}$ potrebbe effettivamente aver avuto come antigrafo un codice molto simile a J, dal momento che tenta di ovviare alla lacuna costituita dall'assenza del terzo metro interpolando (dopo la rubrica che annuncia il m. III) il brano recante la storia di Tiresia, che fa invece parte della terza prosa che precede. Tuttavia non si tratta qui di una dipendenza diretta, dal momento che il modello di $\mathrm{Se}_{1}$ risultava molto probabilmente più completo di J, visto che nella stampa sivigliana i testi della quarta e quinta prosa e del quarto metro risultano completi.

Per J invece, sebbene non si possa escludere del tutto la presenza di una guasto meccanico nell'antigrafo dal quale deriva, guasto per cui le sezioni omesse sarebbero dovute risultare illeggibili (meno probabile la caduta di una carta che potrebbe spiegare il salto dall'inizio della quarta prosa alla fine della quinta, ma non la lacuna tra la fine della terza prosa e l'inizio della quarta), pare più pertinente immaginare - tenendo conto delle proposte avanzate da Zonta e Ziino per il volgarizzamento di Benveniste - che questo codice rappresenti uno stadio iniziale della trasmissione dei materiali aggiunti da Ginebreda al Libro V, qui inseriti solo parzialmente dal copista.

Tuttavia pone a mio avviso un problema ulteriore l'assetto sintattico e lessicale di J che, se presenta un testo la cui struttura e i cui contenti concordano (per i libri I-IV) in linea generale con N, R e $\mathrm{Se}_{1}$, per quel che riguarda la facies sintattica e lessicale $\mathrm{J}$ segue la variante redazionale tràdita dai testimoni di $\beta$. A riguardo può risultare esemplificativa nel dar conto di questa situazione la trascrizione 'sinottica' di tutti i codici ${ }^{23}$ del passo corrispondente a $C P h$ I, 2, 5-6: ${ }^{24}$

23 Indico in tondo le sigle dei testimoni catalani, in corsivo quelle dei testimoni castigliani. V, Y e Z non conservano il passo in esame.

24 «Nihil, inquit, pericli est, lethargum patitur, communem illusarum mentium morbum. Sui paulisper oblitus est. Recordabitur facile, si quidem nos ante cognouerit» (Bieler ed. 1984: 4).

$27 \mathrm{P}$ presenta in questo punto una lacuna dovuta a saut du même au même. 


\section{Chiara Concina. Primi sondaggi sul testimone J del volgarizzamento catalano della Consolatio Philosophiae}

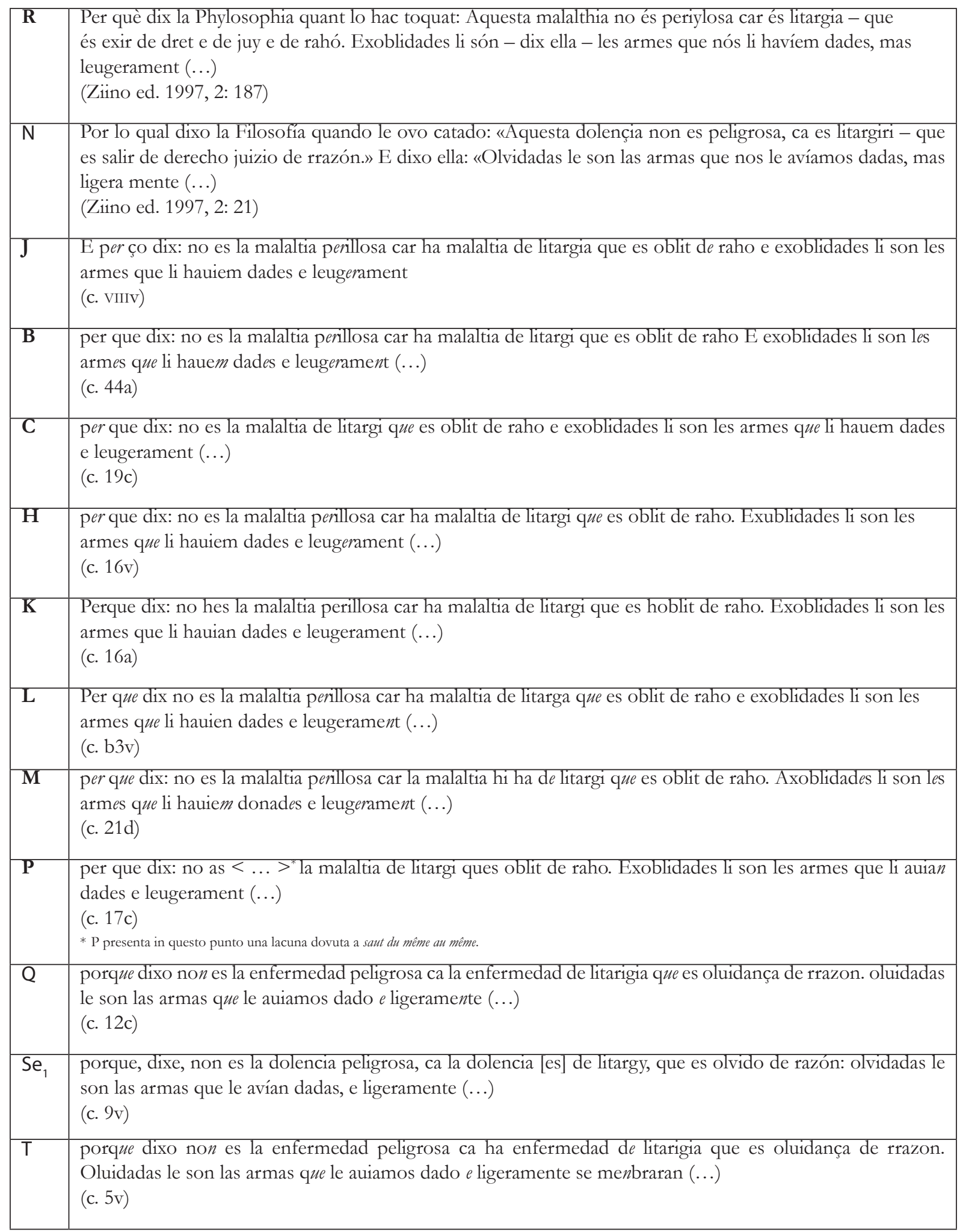


J, B, C, H, K, L, M, P, Q, Se e e T si comportano in modo omogeneo e presentano, rispetto a $\mathrm{R}+\mathrm{N}$, delle semplificazioni, per esempio nell'appiattimento della precisazione «que és exir de dret e de juy e de rahó» di $\mathrm{R}$ (perfettamente sovrapponibile a $\mathrm{N}$ : «que es salir de derecho juizio de rrazón») che viene resa con «que és oblit de rahó»; o ancora nell'eliminazione del sintagma che introduce il discorso diretto di Filosofia (dix ella di R, E dixo ella di N) o nello scorciamento (mediante omissione del soggetto) del periodo posto a introdurre la prima affermazione: «Per què dix la Phylosophia» (R) / «Por lo qual dixo la Filosofía» $(\mathrm{N})$, cui corrispondono le varianti «E per çò dix»/ /per què dix» degli altri testimoni. Tale tipo di dinamica, qui solo accennata, si riscontra costantemente nel confronto tra $\mathrm{R}+\mathrm{N}$ da una parte $\mathrm{e} \mathrm{J}\left(\mathrm{Se}_{1}\right)+\beta$ dall'altra.

Se si ipotizza dunque che $\mathrm{J}$ sia da ricondurre a una prima fase di circolazione e trasmissione di un testo derivante dalla perduta redazione di Saplana, sul quale via via andavano inserendosi i materiali desunti dalla revisione di Ginebreda, si dovrebbe supporre anche che l'assetto sintattico-lessicale del modello di Ginebreda fosse simile a quello di J e che egli si trovò a intervenire su una redazione che, rispetto all'originale, aveva già subíto, nel processo di copiatura, non solo alcune modifiche di forma, ma anche e soprattutto delle perdite proprio in quei punti che egli menziona nel prologo. Questo fatto sarebbe almeno in parte confermato dalle lacune di J, per il quale rimane in ogni caso da spiegare in maniera circostanziata la sua aderenza, per il Libro $\mathrm{V}$, alla redazione $\beta$. Inoltre, in misura maggiore o minore a seconda dei codici, tutta la tradizione del Libro V della Consolatio catalana, presenta, per i latori di $\beta$, una serie di problemi (lacune, interpolazioni, spostamenti) che lasciano intravvedere una situazione di trasmissione problematica e 'perturbata' già all'origine, che necessiterà dunque di ulteriori approfondimenti mediante un'analisi serrata dell'intera tradizione superstite. 
Chiara Concina. Primi sondaggi sul testimone J del volgarizzamento catalano della Consolatio Philosophiae

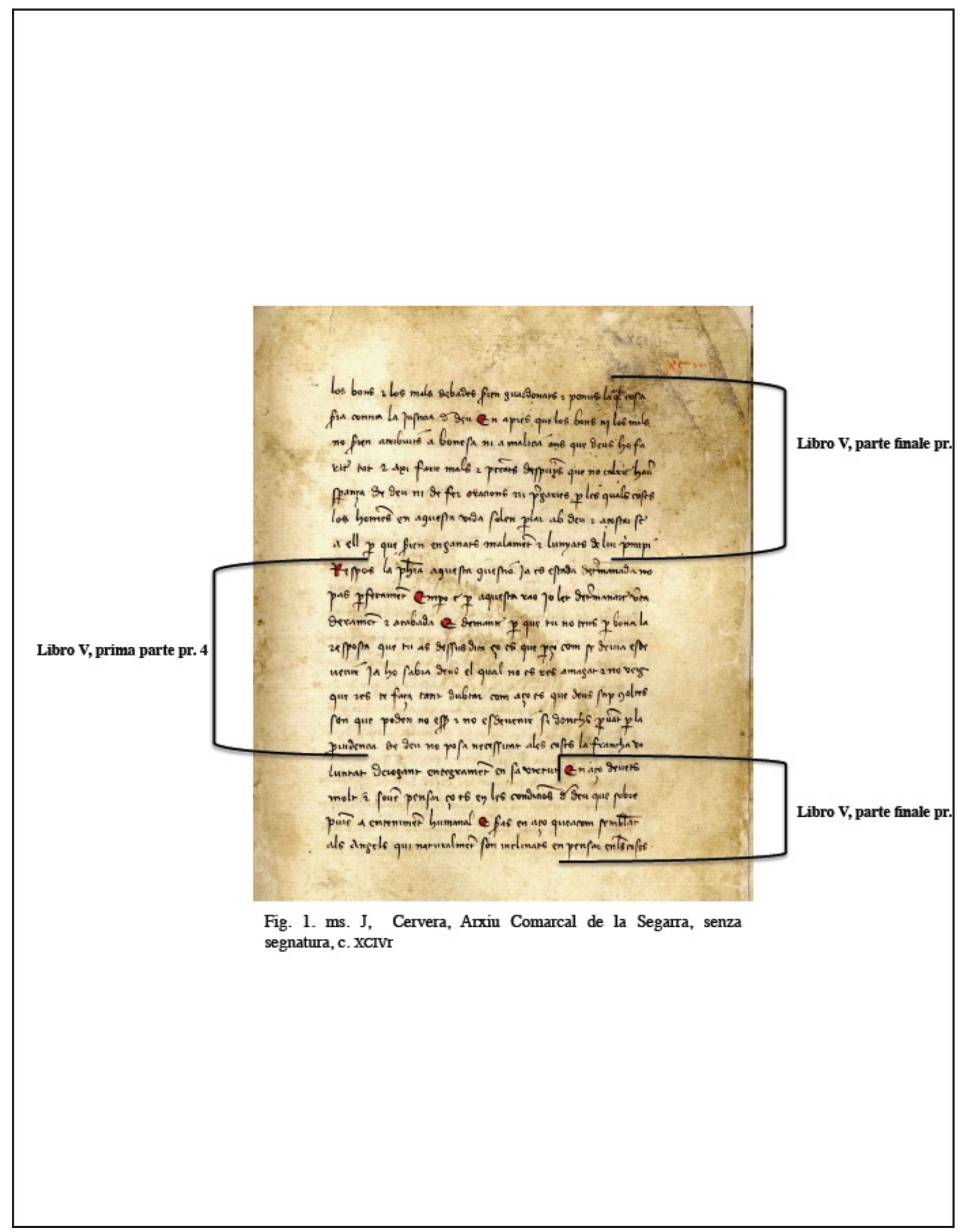

SCRIPTA, Revista internacional de literatura i cultura medieval i moderna, núm. 5 / juny 2015 / pp. 182 - 206 ISSN: 2340-4841 · doi:10.7203/SCRIPTA.5.6390 
Chiara Concina. Primi sondaggi sul testimone J del volgarizzamento catalano della Consolatio Philosophiae

\section{Bibliografia}

Albareda, A. (1919 [1920]) «L'arxiu antic de Montserrat», Analecta Montserratensia, 3, pp. 77-216.

Babbi, A. M. (2010) Saggi sui volgarizzamenti francesi della Consolatio Philosophiae, Verona, Edizioni Fiorini.

Bieler, L. (ed.) (1984) Anicii Manlii Severini Boethii Philosophiae Consolatio, Turnhout, Brepols.

Bofarull y Mascaró, P. (ed.) (1857) Colección de documentos inéditos del Archivo general de la Corona de Aragon. Tomo XIII: Documentos literarios en antigua lengua catalana (Siglos XIV y XV), Barcelona, Imprenta del Archivo.

Coll, J. M. (1935) «Fra Pere Saplana, O.P. autor de la traducció catalana del llibre Consolació de Boeci», La paraula Cristiana, 22, pp. 305-309.

—_. (1949) «Tres escritores dominicos gerundenses del siglo XIV, en lengua vernácula», Annals de l'Institut d'Estudis Gironins, 4, pp. 5-36.

Courcelle, P. (1967) La Consolation de la Philosophie dans la tradition littéraire. Antécédents et postérité de Boèce, Paris, Études Augustiniennes.

Cropp, G. M. (1982-1983) «Les manuscrits du Livre de Boece de Consolacion», Revue d'histoire des textes, 22-23, pp. 263-352.

Doñas, A. (2007) «Versiones hispánicas de la Consolatio Philosophiae de Boecio. Testimonios», Revista de Literatura Medieval, 19, pp. 295-312.

- (ed.) (2004-2005) «La consolación de la Filosofia» de Boecio, en traducción anónima (1497), Alicante, Biblioteca Virtual Miguel de Cervantes, online: http://www.cervantesvirtual.com/nd/ark:/59851/ bmczk5n7.

Galderisi, C. (dir.) (2011) Translations médiévales. Cinq siècles de traductions en français au Moyen Âge. Étude et Répertoire, Turnhout, Brepols, 2 vol., 3 t.

González Rolan, T. / Saquero Suàrez-Somonte, P. (1992) «Boecio en el medioevo hispánico», in Humanitas in honorem Antonio Fontan, Madrid, Gredos, pp. 319-337.

Keightley, R. G. (1987) «Boethius in Spain: A Classified Checklist of Early Translations», in Minnis A. (ed.) The Medieval Boetbius. Studies in the Vernacular Translations of the De Consolatione Philosophiae, Cambridge, D. S. Brewer, pp. 169-187.

Langlois, E. (1896) «Archipiada», in Mélanges de Philologie Romane dédiés à Carl Wablund à l'occasion du cinquantième anniversaire de sa naissance (7 janvier 1896), Mâcon, Protrat Frères.

Muntaner, B. (ed.) (1873-1904) Libre de Consolació de Philosophia lo qual feu en lati lo glorios doctor Boeci, transladat en romanc catalanesch, estampat novament, prol. de À. Aguiló, Barcelona, Llibreria d'Alvar Verdaguer.

Olmedilla Herrero, M. del C. (ed.) (1997) Edición crítica de los comentarios de Guillermo de Aragón al «De 
Chiara Concina. Primi sondaggi sul testimone J del volgarizzamento catalano della Consolatio Philosophiae

Consolatione Philosophiae» de Boecio. Tesis Doctoral, Madrid, Universidad Complutense de Madrid.

Perarnau i Espelt, J. (2006) «El Boeci català complet de Munic (BSB, Hisp. 145)», Arxiu de texts catalans antics, 25, pp. 453-460.

Pérez Rosado, M. (1993) «Dos notas sobre la Consolación de la Filosofía de Boecio en la Edad Media castellana», Cuadernos de Filología Clásica. Estudios latinos, 4, pp. 113-126

Puig i Oliver, J. de (2000) «Alguns documents sobre Antoni Ginebreda, O.P. (1340?-1395)», Arxin de textos catalanos antics, 19, pp. 511-524.

Riera i Sans, J. (1984) «Sobre la difusió hispànica de la Consolació de Boeci», El Crotalón. Anuario de Filología Española, 1, pp. 297-327.

Silk, E. T. (ed.) (1935) Saeculi noni auctoris in Boetii Consolationem Philosophiae commentarius, Rome, American Acedemy in Rome.

(ed.) Expositio Fratris Nicolai Trevethi Anglici Ordinis praedicatorum super Boetio De Consolatione: $<$ http://minnis.commons.yale.edu/>.

Terbille, C. I. (ed.) (1972) William of Aragon's Commentary on Boethius" "De Consolatione Philosophiae», Dissertation, University of Michigan, 2 vol.

Villanueva, J. (1851) Viage literario a las iglesias de España. Tomo XVIII: Viage á Barcelona. Madrid, Imprenta de la Real Academia de la Historia.

Ziino, F. (ed.) (1997) Una versione castigliana del 'De consolatione Philosophiae' (MS Madrid, Biblioteca Nacional, 10193). Tesi di dottorato in Filologia e linguistica romanza, VIII ciclo. Napoli: Università degli Studi di Napoli, 2 vol.

- (1998) «Some Vernacular Versions of Boethius's De Consolatione Philosophiae in Medieval Spain. Notes on their relationship with the Commentary tradition», Carmina Philosophiae, 7, pp. 37-95.

- (2001a) «The Catalan Tradition of Boethius's De consolatione: A New Hypothesis», Carmina Philosophiae. Journal of The International Boethius Society, 10, pp. 31-38.

—_. (2001b) «Una traduzione latina del Boezio catalano», Romania, 119/3-4, pp. 464-482.

—. (2003) «Una traduzione castigliana del De consolatione philosophiae di Boezio (ms. Madrid, Biblioteca Nacional, 10193)», Romanica Vulgaria - Quaderni, 15, pp. 257-273.

_. (2007) «Some Vernacular Versions of Boethius’s De Consolatione Philosophiae in Medieval Spain. Notes on their relationship with the Commentary tradition» in Kaylor, N. H. Jr. / Phillips, P. E. (eds.) New Directions in Boethian Studies, Kalamazoo (Michigan), Western Michigan University, 2007, pp. 83-107 (rist. ampliata di Ziino 1998).

_ . (2010) «The Catalan Tradition of Boethius’s De consolatione: A New Hypothesis», in Glei, R. F. / Kaminski, N. / Lebsanft, F. (eds.) Boethius Christianus? Transformationen der Consolatio Philosophiae in Mittelalter und Früher Neuzeit, Berlin, De Gruyter, pp. 387-395 (rist. ampliata di Ziino 2001a).

Zonta, M. (2010) «Le origini letterarie e filosofiche delle versioni ebraiche del De Consolatione Philosophiae di Boezio», in Glei, R. F. / Kaminski, N. / Lebsanft, F. (eds.) Boethius Christianus? Transformationen der Consolatio Philosophiae in Mittelalter und Früher Nenzeit, Berlin, De Gruyter, pp. 397-429.

SCRIPTA, Revista internacional de literatura i cultura medieval i moderna, núm. 5 / juny 2015 / pp. 182 - 206 ISSN: 2340-4841 · doi:10.7203/SCRIPTA.5.6390 